\title{
A Kochen-Specker system has at least 22 vectors
}

\author{
(extended abstract)*
}

\author{
Sander Uijlen \\ Radboud Universiteit \\ suijlen@cs.ru.nl
}

\author{
Bas Westerbaan \\ Radboud Universiteit \\ bwesterb@cs.ru.nl
}

\begin{abstract}
At the heart of the Conway-Kochen Free Will Theorem and Kochen and Specker's argument against non-contextual hidden variable theories is the existence of a Kochen-Specker (KS) system: a set of points on the sphere that has no $\{0,1\}$-coloring such that at most one of two orthogonal points are colored 1 and of three pairwise orthogonal points exactly one is colored 1. In public lectures, Conway encouraged the search for small KS systems. At the time of writing, the smallest known KS system has 31 vectors.

Arends, Ouaknine and Wampler have shown that a KS system has at least 18 vectors, by reducing the problem to the existence of graphs with a topological embeddability and non-colorability property. The bottleneck in their search proved to be the sheer number of graphs on more than 17 vertices and deciding embeddability.

Continuing their effort, we prove a restriction on the class of graphs we need to consider and develop a more practical decision procedure for embeddability to improve the lower bound to 22 .
\end{abstract}

\section{Introduction}

\subsection{The experiment}

Consider the following experiment. Shoot a deuterium atom (or another neutral spin 1 particle) through a certain fixed inhomogeneous magnetic field, such as that in the Stern-Gerlach experiment. The particle will then move undisturbed or deviate. What we have done is measure the spin componen 11 of the particle along a certain direction. This direction depends on the specifics of the field and the movement of the particle.

Quantum Mechanics only predicts the probability, given the direction, whether the particle will deviate. Its probabilistic prediction has been thoroughly tested. One wonders: is there a deterministic theory predicting the outcome of this experiment?

Kochen and Specker have shown that such a non-contextual deterministic theory must be odd: it cannot satisfy the plausible SPIN axiom, that is:

SPIN Axiom [5]. Given three pairwise orthogonal directions. In exactly one of the directions, the particle will not deviate.

Their argument is based on the existence of a Kochen-Specker system.

${ }^{*}$ This is a condensed version of the article to be published in Ohmsha-Springer's New Generation Computing. One can find a preprint of the full version at http://westerbaan. name/ bas/math/ks.pdf

${ }^{1}$ As we are only interested in whether the particle deviates or not, we actually only consider the square of the spin component.

B. Coecke, I. Hasuo \& P. Panangaden (Eds.): Quantum Physics and Logic 2014 (QPL 2014).

EPTCS 172, 2014, pp. 154 164 doi 10.4204/EPTCS.172.11 (c) Uijlen \& Westerbaan

This work is licensed under the Creative Commons Attribution License. 
Definition 1. A Kochen-Specker (KS) system is a finite set of points on the sphere 2 for which each pair is not antipodal and there is no 010-coloring. A 010 -coloring is a $\{0,1\}$-coloring of the points such that

1. no pair of orthogonal points are both colored 1 and

2. of three pairwise orthogonal points exactly one is colored 1 ; or alternatively: they are colored 0,1 and 0 in some order.

A point on the sphere obviously corresponds to a direction in space. Because of this, the term point, vector and direction can be used interchangeably. Antipodal points correspond to opposite vectors and these span the same direction in space.

Suppose there is a KS system and a non-contextual deterministic theory satisfying the SPIN Axiom. Then we color a point of this system 0 , whenever this theory predicts that the particle will deviate if the spin is measured in the direction corresponding to that point, and 1 otherwise. Given two orthogonal points of the system, we can find a third point orthogonal to both of them. The SPIN axiom implies exactly one of them is colored 1, so they cannot both be colored 1. Similarly, given three pairwise orthogonal vectors in the system, the SPIN axiom implies exactly one of them is colored 1 . Hence there would be a 010-coloring of the KS system, quod non. Therefore a deterministic non-contextual theory cannot satisfy the SPIN Axiom.

The KS system proposed by Kochen and Specker contained 117 points[7]. Penrose and Peres[10] independently found a smaller system of 33 points. The current record is the 31 point system of Conway[11,

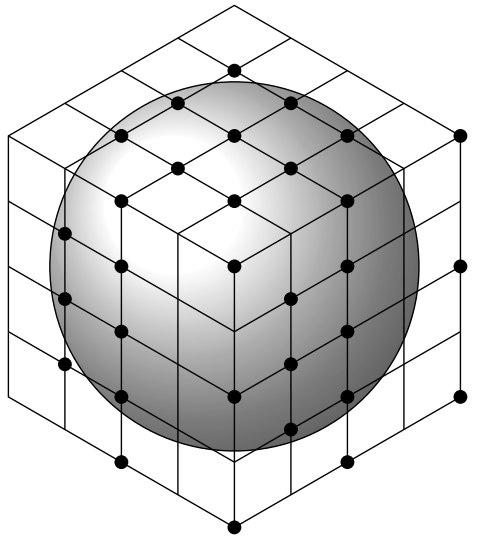

Figure 1: Conway's 31 vector Kochen-Specker system p. 197]. As pointed out by [3, 2], finding small KS systems is of both theoretical and practical interest. In public lectures, Conway himself, stressed the search for small KS systems.[9]

\subsection{Overview}

In [2] Arends, Ouaknine and Wampler (AOW) give a computer aided proof that a KS system must have at least 18 vectors. We improve their lower bound and show that a KS system must have at least 22 vectors.

First, in Subsection 1.3, we repeat a part of AOW's work, in particular the reduction of KS systems to graphs. The bottleneck of their search was the sheer number of graphs and the deciding whether such graphs are embeddable. In Section 2 , we improve upon their reduction, to cut down the number of graphs to consider drastically, and state the results of our main computation. Finally, in Section 3, we describe our practical embeddability test.

The software and results of the various computations performed for this paper, can be found here[15].

\subsection{Kochen-Specker graphs}

We follow [2] and reduce the search for Kochen-Specker systems to the search of a certain class of graphs. First note that in a Kochen-Specker system we may replace a point with its antipodal point. They

\footnotetext{
${ }^{2}$ We define KS systems to be three dimensional, as in the original proof of Kochen and Specker. Later, higher dimensional systems have been studied. See, for instance [11 p. 201].

${ }^{3}$ In other papers, like [2], the 0 and 1 are swapped; they consider 101-colorings. These colorings are of course equivalent and the difference arises from considering either squared spin measurements $S^{2}$, or $1-S^{2}$.
} 
are both orthogonal to the same points and hence the non-010-colorability is preserved. Therefore, we may assume antipodal points are identified on the sphere. That is: a Kochen-Specker system is a finite subset of the projective plane that is not 010-colorable.

Definition 2. Given a finite subset $S$ of the projective plane (or equivalently, a finite subset of the northern hemisphere without equator $\left.{ }^{4}\right)$. Define its orthogonality graph $G(S)$ as follows. The vertices are the points of $S$. Two vertices are joined by an edge, if their corresponding points are orthogonal.

Definition 3. A graph $G$ is called embeddable, if it occurs as a subgraph of an orthogonality graph. That is: if there is a finite subset $S$ of the projective plane, such that $G \leq G(S)$.

Definition 4. A graph is called 010-colorable if there is a $\{0,1\}$-coloring of the vertices, such that

1. for each triangle there is exactly one vertex that is colored 1 and

2. adjacent vertices are not both colored 1 .

Definition 5. A Kochen-Specker graph is a embeddable graph that is not 010-colorable.

It is an easy, but important, consequence of the definitions that:

Fact 6. A finite subset $S$ of the projective plane is a Kochen-Specker system, if and only if its orthogonality graph $G(S)$ is Kochen-Specker.

To prove there is no Kochen-Specker system on 17 points, it would be sufficient to enumerate all graphs on 17 vertices and check these are not 010-colorable or not embeddable. However, this is infeasible as there are already $\sim 10^{26}$ non-isomorphic graphs on 17 points.[12] Luckily, we can restrict ourselves to certain classes of graphs.

Proposition 7 ([2]). An embeddable graph is squarefree. That is: it does not contain the square as a subgraph:5

Proof. Given two non antipodal points $v \neq w$. See the figure on the right. Consider the points orthogonal to $v$. This is a great circle. The points orthogonal to $w$ is a different great circle. They intersect in precisely two antipodal points. Hence, if $c$ and $d$ are both orthogonal to $v$ and $w$, then $c$ and $d$ are equivalent. Therefore, an embeddable graph cannot contain a square.

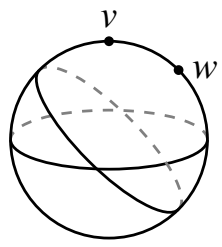

The squarefreeness is a considerable restriction. There are only $\sim 10^{10}$ non-isomorphic squarefree graphs on 17 vertices.[13] Next, we show we can restrict ourselves to connected graphs.

Proposition 8 ([2]). A minimal Kochen-Specker graph is connected.

Proof. Suppose $G$ is a non-connected Kochen-Specker graph. Then one of its components is not 010colorable. As a subgraph of an embeddable graph, is embeddable, this component is embeddable as well. Hence it is a smaller connected Kochen-Specker graph.

The gain, however, is small. There are only $\sim 10^{9}$ non-isomorphic squarefree graphs on 17 vertices that are not connected. In our computations, checking for connectedness required more time than would be gained by reducing the number of graphs.

We have verified the main result of [2]:

\footnotetext{
${ }^{4} \mathrm{~A}$ subset of the projective plane can be identified with a subset of the closed northern hemisphere. For a finite subset we can always rotate in such a way that no points lie on the equator.

${ }^{5}$ Some authors call a graph squarefree if it does not contain the square as induced subgraph. For them the complete graph on four vertices is squarefree. We follow Weisstein[16] and Sloane[13] and call a graph squarefree if it does not contain the square as subgraph. For us the complete graph on four vertices is not squarefree.
} 
Computation 9. There is a unique non-010-colorable squarefree connected graph on 17 or less vertices:

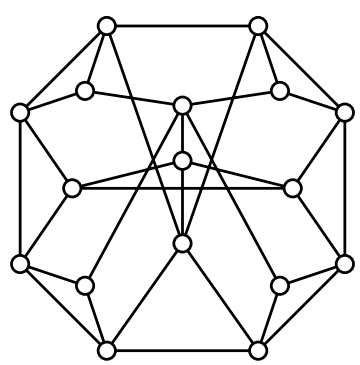

It is not embeddable, as the graph in Figure 2 is an unembeddable subgraph. For our proof, see Proposition 22 Hence a Kochen-Specker system has at least 18 points.

\section{An improved lower bound}

Continuing the effort of Arends, Ouaknine and Wambler, we consider another restriction.

Proposition 10. A minimal Kochen-Specker graph has minimal vertex-order three. That is: every vertex is adjacent to at least three other vertices.

Proof. Given a minimal Kochen-Specker graph G. Suppose $v$ is a vertex with order less than or equal 2. Let $G^{\prime}$ be $G$ with $v$ removed. Clearly $G^{\prime}$ is embeddable. Suppose $G^{\prime}$ is 010 -colorable. Then we can extend the coloring to a coloring of $G$ as follows. If $v$ is adjacent to only one or no vertex, then we can color $v$ with 0 . Suppose $v$ is adjacent to two vertices, say $w$ and $w^{\prime}$. If one of $w$ or $w^{\prime}$ is colored 1 , we can color $v$ with 0 . If both $w$ and $w^{\prime}$ are colored 0 , we can color $v$ with 1 . This would imply $G$ is 010 -colorable, quod non. Therefore $G^{\prime}$ is a smaller Kochen-Specker graph, which contradicts the minimality of $G$.

There are only $\sim 10^{7}$ squarefree non-isomorphic graphs on 17 vertices with minimal vertex order 3 . Even though Arends, Ouaknine and Wampler note this restriction once, surprisingly, they did not restrict their graph enumeration to graphs with minimal vertex order 3.

We continue with a strengthening of Proposition 8 .

Proposition 11. A minimal Kochen-Specker graph is edge-biconnected. That is: removing any single edge leaves the graph connected.

We need some preparation, before we can prove this Proposition.

Definition 12. Given a graph $G$ and a vertex $v$ of $G$. We say, $v$ has fixed color $c$ (in $G$ ), if $G$ is 010 colorable and for every 010 -coloring of $G$, the vertex $v$ is assigned color $c$.

We are interested in these graphs because of the following observation.

Lemma 13. If there is an embeddable graph $G$ on $n$ vertices with a vertex with fixed color 1 , then there is a Kochen-Specker graph on $2 n$ vertices.

Proof. Let $G$ be a graph and $v$ a vertex of $G$ with fixed color 1. Consider two copies of the graph $G$. Connect the two instances of $v$ with an edge. Call this graph $G^{\prime}$. Clearly, $G^{\prime}$ is not 010-colorable.

We need to show $G^{\prime}$ is embeddable. Given an embedding $S$ of $G$. We may assume that the point in $S$ corresponding to $v$ is the north pole. Furthermore, we may assume that there is no point on the $x$-axis, by rotating points along the north pole. Let $S^{\prime}$ be $S$ rotated 90 degrees along the $y$-axis. Some points 
of $S$ and $S^{\prime}$ might overlap. That is: there might be a point $s$ in $S$ and $s^{\prime}$ in $S^{\prime}$ that are equal or antipodal. Observe that if no points of $S^{\prime}$ and $S$ overlap, then $S \cup S^{\prime}$ is an embedding of $G^{\prime}$.

Suppose there are points in $S^{\prime}$ and $S$ that overlap. Note that the north pole (and south pole) is not in $S^{\prime}$. Let $S^{\prime \prime}$ be $S^{\prime}$ rotated along the north pole at some angle $\alpha$. There are finitely many angles such that there are overlapping points. Thus there is an angle such that $S \cup S^{\prime \prime}$ is an embedding of $G^{\prime}$.

Unfortunately, these graphs are not small.

Computation 14. There are no embeddable graphs with fixed color 1 on less than 17 vertices.6

We are ready to prove that a minimal Kochen-Specker graph is edge-biconnected.

Proof of Proposition 11] Given a minimal Kochen-Specker graph G.

Recall it must be connected. Suppose it is not edge-biconnected. Then there must be an edge $(a, b)$ in $G$, which removal disconnects $G$. Thus $G$ decomposes into two connected graphs $A$ and $B$ such that $a \in A, b \in B$ and $(a, b)$ is the only edge between $A$ and $B$. Clearly $A$ and $B$ are embeddable.

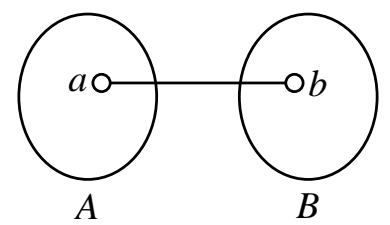

Note that $A$ must be 010-colorable, for if it were not 010-colorable, then $A$ is a Kochen-Specker graph, in contradiction with $G$ 's minimality. Similarly $B$ is 010 -colorable. Suppose there is a 010 -coloring of $A$ in which $a$ is colored 0 . Then we can extend this coloring with any 010-coloring of $B$ to a 010-coloring of $G$, which is absurd. Thus $a$ must have fixed color 1 in $A$. Similarly $b$ must have fixed color 1 in $B$. Thus by Computation 14 , we have $\# A \geq 17$ and $\# B \geq 17$. Consequently $\# G \geq 34$. Contradiction with $G$ 's minimality.

We can go one step further.

Proposition 15. A minimal Kochen-Specker graph is edge-triconnected. That is: removing any two edges keeps the graph connected.

Again, we need some preparation for the proof. First, we generalize the notion of fixed color.

Definition 16. Given a graph $G$ together with selected vertices $v_{1}, \ldots, v_{n} \in G$. Let $C(G)$ denote the set of 010-colorings of $G$. The type $t$ of $\left(v_{1}, \ldots, v_{n}\right)$ (in $G$ ) is the set of all possible ways $\left\{v_{1}, \ldots, v_{n}\right\}$ can be colored. That is: $t=\left\{\left(c\left(v_{1}\right), \ldots, c\left(v_{n}\right)\right) ; c \in C(G)\right\}$. A type of $n$ vertices is called an $n$-type.

Example 17. - The triangle has 3-type $\{(1,0,0),(0,1,0),(0,0,1)\}$.

- Every vertex in a Kochen-Specker graph has type $\emptyset$.

- A vertex $v$ has the 1-type $\{(1)\}$ in $G$ if and only if it has fixed color 1 in $G$.

Just as verteces with fixed color are rare, we are interested in types, because most types do not occur in small graphs.

Computation 18. We have enumerated all embeddable graphs of less than 17 vertices and determined a lower bound at which a particular 1- or 2-type occurs, omitting the trivial types $\{(0),(1)\}$ and $\{(0,0),(0,1),(1,0),(1,1)\}$.7

\footnotetext{
${ }^{6}$ Source code at code/comp5. py of [15].

${ }^{7}$ Source code at code/comp5. py of [15].
} 


\begin{tabular}{ll} 
1/2-type & $\# G$ \\
\hline$\{(0,0),(1,0),(0,1)\}$ & non-trivially $\geq 10$ \\
$\{(0,0),(1,0),(1,1)\}$ & $\geq 10$ \\
$\{(0,0),(0,1),(1,1)\}$ & $\geq 10$ \\
$\{(0,0),(0,1))\}$ & $\geq 15$ \\
$\{(0,0),(1,0))\}$ & $\geq 15$ \\
$\{(0)\}$ & $\geq 15$ \\
$\{(0,1),(1,0)\}$ & $\geq 16$ \\
other & $\geq 17$
\end{tabular}

The type $\{(0,0),(1,0),(0,1)\}$ occurs in the embeddable two-vertex graph $\mathrm{0}-\mathrm{O}$. Because the two vertices are adjacent, this occurance of the type is called trivial.

Proof of Proposition 15 Given a minimal Kochen-Specker graph G.

Suppose it is not edge-triconnected. Then it splits into two graphs $A$ and $B$ together with verteces $a_{1}, a_{2} \in A$ and $b_{1}, b_{2} \in B$ such that $\left(a_{1}, b_{1}\right)$ and $\left(a_{2}, b_{2}\right)$ are the only edges between $A$ and $B$. Note that $A$ and $B$ must be 010 -colorable, for otherwise $G$ would not be a minimal Kochen-Specker graph.

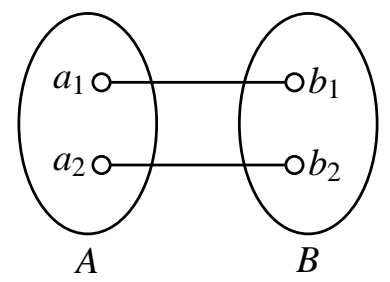

1. Suppose $a_{1}=a_{2}$ and $b_{1}=b_{2}$. Then $G$ is not edge-biconnected. Contradiction with Proposition 11 .

2. Suppose $a_{1} \neq a_{2}$ and $b_{1}=b_{2}$. Suppose $b_{1}=b_{2}$ does not have a fixed color in $B$. Then any coloring of $A$ can be extended with some coloring in $B$ to a coloring of $G$. Contradiction. Apparently $b_{1}=b_{2}$ has a fixed color in $B$.

(a) Suppose $b_{1}=b_{2}$ has fixed color 1 in $B$. Note $\# B \geq 17$ by Computation 18

Suppose there is a coloring of $A$ in which both $a_{1}$ and $a_{2}$ have color 0 . Then, regardless whether $a_{1}$ and $a_{2}$ are adjacent or not, this coloring can be extended with a coloring of $B$ (in which $b_{1}=b_{2}$ must be colored 1$)$ to a coloring $G$. Contradiction.

Thus the type of $\left(a_{1}, a_{2}\right)$ in $A$ cannot contain $(0,0)$. Thus, by Computation 18, $\# A \geq 17$. Consequently $\# G \geq 34$. Contradiction with minimality.

(b) Apparently $b_{1}=b_{2}$ has fixed color 0 in $B$. Hence, by Computation 18 , $\# B \geq 15$.

Suppose $a_{1}$ is not adjacent to $a_{2}$. Then any coloring of $A$ can be extended with a coloring of $B$ to a coloring of $G$. Contradiction.

Apparently $a_{1}$ is adjacent to $a_{2}$.

The type of $\left(a_{1}, a_{2}\right)$ in $A$ cannot contain $(1,0)$ or $(0,1)$ for otherwise $G$ can be colored. It also cannot contain $(1,1)$ as $a_{1}$ and $a_{2}$ are adjacent. Thus both $a_{1}$ and $a_{2}$ have fixed color 0 in $A$. Hence $\# A \geq 17$ by Computation 18 . Consequently $\# G \geq 32$. Contradiction with minimality.

3. Suppose $a_{1}=a_{2}$ and $b_{1} \neq b_{2}$. This leads to a contradication in the same way as in case 2 ,

4. Apparently $a_{1} \neq a_{2}$ and $b_{1} \neq b_{2}$. The type of $\left(a_{1}, a_{2}\right)$ in $A$ cannot contain $(0,0)$, for otherwise $G$ is colorable. Similarly, the type of $\left(b_{1}, b_{2}\right)$ in $B$ cannot contain $(0,0)$. Thus both $\# A \geq 17$ and $\# B \geq 17$. Hence $\# G \geq 34$. Contradiction with minimality.

Although these restrictions are theoretically pleasing, they seem to be of little use as a practical restriction. Concerning excluding unconnected graphs:

Computation 19. There are five non-isomorphic minimal squarefree connected graphs with minimal vertex order 3 and they have 10 vertices. 
Corollary 20. Any unconnected squarefree graph with minimal vertex order 3 has at least 20 vertices, for it has two connected components, each with at least 10 vertices. With 20 vertices, there are exactly 25 of these.

This justifies, at this stage, not checking for connectedness. Similarly, we believe there are very few connected but not edge-biconnected graphs.

Now we can state our main computation.

Computation 21. Let $C_{n}$ denote the number of non-010 colorable squarefree graphs with minimal vertex order 3 on $n$ nodes. Then: 8

\begin{tabular}{l|llllll}
$n$ & $\leq 16$ & 17 & 18 & 19 & 20 & 21 \\
\hline$C_{n}$ & 0 & 1 & 2 & 19 & 441 & 11876
\end{tabular}

All these 12339 graphs are not embeddable. See Computation 23.

The computation was distributed on approximately $300 \mathrm{CPU}$ cores and took roughly three months. It was executed as follows. We enumerated all squarefree graphs with minimal vertex order 3 on less than or equal 21 vertices, using the geng util of the nauty software package, which uses the isomorphism-free exhaustive generation method of McKay[8]. The output of geng, we passed through a custom heuristic backtracker written in $\mathrm{C}++$ to decide 010 -colorability of these graphs.

\section{Embeddability}

Our computation has yielded over nine-thousand non-010-colorable graphs. If we show one of them is embeddable, we have found a new KS system. If we demonstrate all of them are not embeddable, we have proven a lower bound on the size of a minimal KS system.

In [2], Arends, Wampler and Ouaknine discuss several computer-aided methods to test embeddability of a graph. None of these methods could decide for all graphs considered, whether they were embeddable or not.

We propose a new method, which for all graphs we considered, could decide whether they were embeddable or not. First we give a pen-and-paper example.

Proposition 22. The graph in Figure 2 is not embeddable.

Proof. Suppose it is embeddable. Consider $p_{1}$. It is orthogonal to both $a$ and $v$. Since $a$ and $v$ are not collinear, $p_{1}$ must be collinear to $v \times a$, the cross-product of $v$ and $a$. Similarly, $p_{2}$ is collinear to $v \times$ $p_{1}=v \times(v \times a)$. Continuing in this fashion, we see that

$$
a \text { is collinear to } x \times(x \times(w \times(w \times(v \times(v \times a))))) \text {. }
$$

Now, we may assume that $z=(0,0,1)$ and $x=(1,0,0)$. Thus: $v=$

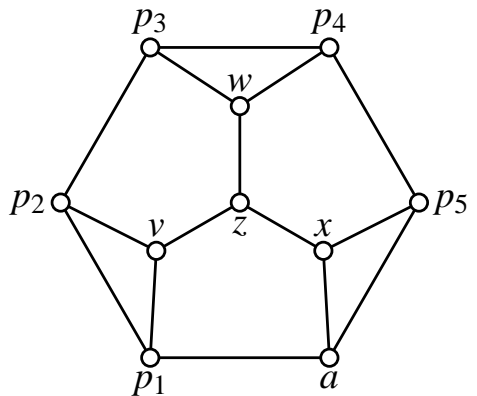

Figure 2: One of the two minimal non-embeddable graphs $\left(v_{1}, v_{2}, 0\right) ; w=\left(w_{1}, w_{2}, 0\right)$ and $a=\left(0, a_{2}, a_{3}\right)$ for some $-1 \leq v_{1}, v_{2}, w_{1}, w_{2}, a_{2}, a_{3} \leq 1$, with $v_{1}^{2}+v_{2}^{2}=1$; $w_{1}^{2}+w_{2}^{2}=1$ and $a_{2}^{2}+a_{3}^{2}=1$. Now, (1) becomes:

$$
\left(\begin{array}{c}
0 \\
a_{2} \\
a_{3}
\end{array}\right) \text { is collinear to }\left(\begin{array}{c}
0 \\
-a_{2} v_{1} w_{2}\left(v_{1} w_{1}+v_{2} w_{2}\right) \\
-a_{3}\left(v_{1}^{2} w_{1}^{2}+v_{1}^{2} w_{2}^{2}+v_{2}^{2} w_{1}^{2}+v_{2}^{2} w_{2}^{2}\right)
\end{array}\right) \text {. }
$$

\footnotetext{
${ }^{8}$ Source code at code/comp6 of [15].
} 
Consequently

$$
\begin{aligned}
v_{1} w_{2}\left(v_{1} w_{1}+v_{2} w_{2}\right) & =v_{1}^{2} w_{1}^{2}+v_{1}^{2} w_{2}^{2}+v_{2}^{2} w_{1}^{2}+v_{2}^{2} w_{2}^{2} \\
& =\left(v_{1}^{2}+v_{2}^{2}\right) w_{1}^{2}+\left(v_{1}^{2}+v_{2}^{2}\right) w_{2}^{2} \\
& =w_{1}^{2}+w_{2}^{2} \\
& =1 .
\end{aligned}
$$

Since $v$ and $w$ are not collinear, we have by Cauchy-Schwarz $|\langle v, w\rangle|<1$. Now we find the contradiction:

$$
1>\left|v_{1} w_{2}\langle v, w\rangle\right|=\left|v_{1} w_{2}\left(v_{1} w_{1}+v_{2} w_{2}\right)\right|=1 .
$$

In the previous proof, we fixed, without loss of generality, the position of a few vertices. Then we derived cross-product expressions for the remaining vertices. Finally, we find an equation relating some of the cross-product expressions and show it is unsatisfiable. We can automate this reasoning as follows.

while there are unassigned vertices do

pick an unassigned vertex $v$

assign $V(v)=v$

mark $v$ as free

5: $\quad$ while there are unassigned vertices adjacent to two different assigned vertices do pick such a vertex $w$ adjacent to the assigned $w_{1}$ and $w_{2}$ assign $V(w)=V\left(w_{1}\right) \times V\left(w_{2}\right)$

mark edges $\left(v, w_{1}\right)$ and $\left(v, w_{2}\right)$ as accounted for

end while

10: end while

for each pair of vertices $\left(v_{1}, v_{2}\right)$ do

if $\left(v_{1}, v_{2}\right)$ is not an edge then

| record requirement: " $V\left(v_{1}\right)$ is not collinear to $V\left(v_{2}\right)$ "

end if

15: end for

for each edge $\left(v_{1}, v_{2}\right)$ not accounted for do

| record requirement: " $V\left(v_{1}\right)$ is orthogonal to $V\left(v_{2}\right)$ "

end for

At two points in the algorithm, there is a choice which vertex to pick. Depending on the vertices chosen, the number of recorded requirements and free points may significantly vary. By considering all possible choices, one can find the one with least free points.

The requirements can be mechanically converted to a formal sentence in the language of the real numbers. This sentence is true if and only if the graph is embeddable. Famously, Tarski proved[14] that such sentences are decidable. His decision procedure has an impractical complexity. However, its practical value has been improved by, for instance, the method of cylindrical algebraic decomposition [4]. We have used the redlog[6] package of the reduce algebra system, which implements a variant of Tarski's quantifier elimination 9

Different assignments give different sentences. In our tests, some assignments would yield sentences that were decided within milliseconds, whereas another assignment with less free vertices would yield

\footnotetext{
${ }^{9}$ The reader can find the reduce script generated mechanically for the graph in Figure 2 here: http://kochen-specker . info/smallGraphs/49743f49514769444f.html.
} 
a sentence that could not be decided (directly). Therefore, when determining embeddability of a graph, we try several assignments in parallel.

In this way, there were still a few (010-colorable) graphs of which we could not decide embeddability. With some guessing, we determined embeddings for these graphs by hand. Once we knew the troublesome graphs were embeddable, we adapted the algorithm, as to guess for some assignments the position of one of the vectors. If the corresponding sentence turns out false, we know nothing. However, if the sentence is true, we know the graph is embeddable.

With this method, we have decided in a day the embeddability of every squarefree graph with minimal vertex order three of less than 15 , except for one 10 In particular:

Computation 23. Every squarefree graph of minimal vertex order three that is not 010-colorable of order less than or equal to 20 contains, as a subgraph, one of the following three graphs:
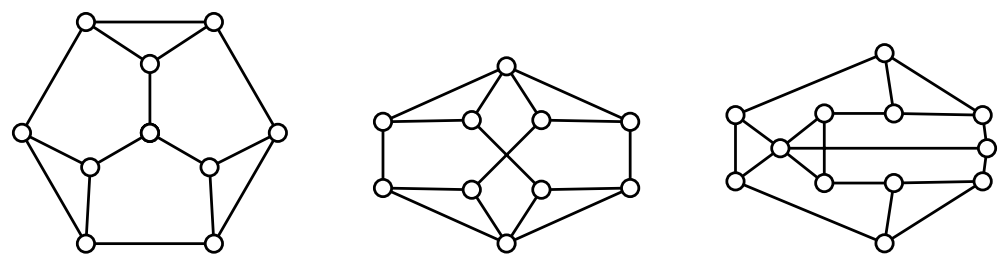

These three graphs are unembeddable. The left and middle graph are the only minimal unembeddable squarefree graph. For the first graph, we have proven directly that it is unembeddable. See Proposition 22. For the second graph, we also have a similar direct proof. The third graph is shown to not be embeddable using our algorithm.

Every squarefree graph of minimal vertex order three that is not 010-colorable of order 21 contains an unembeddable subgraph.11

\section{Conclusion and future research}

Arends, Ouaknine and Wampler struggled with two problems: enumerating candidate graphs of less than 31 vertices and testing their embeddability. We have verified most of their computations. Then we enumerated all candidate graphs up to and including 21 vertices. Furthermore, we have proposed a new decision procedure, which was able to decide embeddability for all candidate graphs we found. Therefore, we demonstrate: a Kochen-Specker system must have at least 22 points 12

Enumerating all candidate graphs of less than 31 vertices is computationally infeasable. To bridge the enormous the gap between 22 and 31, requires a new insight. For instance: another restriction on which graphs to consider.

The Reader, interested in pursuing this line of research, is encouraged to read the master thesis[1] of Arends, in which he discusses in detail several other properties that a minimal KS system must enjoy, as well as some failed attempts.

\footnotetext{
${ }^{10}$ A list of all squarefree graphs with minimal vertex order three of less than 15 vertices together with their embeddability can be found here: http://kochen-specker. info/smallGraphs/. The graph for which we could not determine embeddability can be found here: http://kochen-specker. info/smallGraphs/4d4b3f4b3f603f47414641654953625f3f.html.

${ }^{11} \mathrm{~A}$ list of these graphs together with their unembeddable subgraphs, can be found here: http://kochen-specker.info/ candidates/. The source code for this computation can be found at code/comp2.py of [15].

${ }^{12}$ The authors have a wager whether there is a minimal KS system of less than 25 vertices.
} 


\section{Acknowledgments}

We wish to thank the following for their generous contribution to the distributed computation: the Digital Security group, Intelligent Systems group and the C\&CZ service of the Radboud University; Wouter Geraedts and Jille Timmermans.

We are grateful to prof. McKay for discussing the feasibility of certain graph restrictions and to Judith van Stegeren 13 for drawing figures for the draft version of this paper.

\footnotetext{
${ }^{13}$ http://jd7h.com/
} 


\section{References}

[1] Felix Arends (2009): A Lower Bound on the Size of the Smallest Kochen-Specker Vector System in Three Dimensions. Master's thesis, University of Oxford. Available at http://www.cs.ox.ac.uk/people/ joel.ouaknine/download/arends09.pdf.

[2] Felix Arends, Joël Ouaknine \& Charles W Wampler (2011): On searching for Small Kochen-Specker Vector Systems. In: Proceedings of the 37th international conference on Graph-Theoretic Concepts in Computer Science, Springer-Verlag, pp. 23-34, doi:10.1007/978-3-642-25870-1_4.

[3] Adán Cabello (2000): Kochen-Specker theorem and experimental test on hidden variables. International Journal of Modern Physics A 15(18), pp. 2813-2820, doi:10.1016/S0217-751X (00) 00202-9.

[4] George E Collins (1998): Quantifier elimination for real closed fields by cylindrical algebraic decomposition. In: Quantifier elimination and cylindrical algebraic decomposition, Springer, pp. 85-121, doi:10.1007/ 978-3-7091-9459-1_2.

[5] John H Conway \& Simon Kochen (2009): The strong free will theorem. Notices of the AMS 56(2), pp. 226-232.

[6] Andreas Dolzmann \& Thomas Sturm (1997): Redlog: Computer algebra meets computer logic. Acm Sigsam Bulletin 31(2), pp. 2-9, doi:10.1145/261320.261324.

[7] Simon Kochen \& EP Specker (1975): The problem of hidden variables in quantum mechanics. In: The Logico-Algebraic Approach to Quantum Mechanics, Springer, pp. 293-328, doi:10.1007/ 978-94-010-1795-4_17.

[8] Brendan D McKay (1998): Isomorph-free exhaustive generation. Journal of Algorithms 26(2), pp. 306-324, doi:10.1006/jagm.1997.0898.

[9] Joël Ouaknine: personal communication. Attended such a lecture of Conway at the Oxford Mathematical Institute in 2005.

[10] Asher Peres (1991): Two simple proofs of the Kochen-Specker theorem. Journal of Physics A: Mathematical and General 24(4), p. L175, doi:10.1088/0305-4470/24/4/003.

[11] Asher Peres (1995): Quantum theory: concepts and methods. 57, Springer.

[12] N. J. A. Sloane: The On-Line Encyclopedia of Integer Sequences. Available at http://oeis .org/A000088. Number of graphs on $\mathrm{n}$ unlabeled nodes.

[13] N. J. A. Sloane: The On-Line Encyclopedia of Integer Sequences. Available at http://oeis . org/A006786. Squarefree graphs on $\mathrm{n}$ vertices.

[14] Alfred Tarski (1998): A decision method for elementary algebra and geometry. Springer.

[15] Sander Uijlen \& Bas Westerbaan: Code and Data for "A Kochen-Specker system has at least 22 vectors", doi:10.5281/zenodo.11718. Available at https://github.com/bwesterb/ks.

[16] Eric W. Weisstein: Square-Free Graph. Available at http://mathworld.wolfram.com/ Square-FreeGraph.html. Last visited on may 6th 2014. 